\title{
Conservación en la ciudad: ¿Cómo influye la estructura del hábitat sobre la abundancia de especies de aves en una metrópoli latinoamericana?
}

\section{Conservation in the city: How does habitat structure influence the abundance of individual bird species in a Latin American metropolis?}

\author{
Javiera F. Benito¹, Martín A. H. Escobar ${ }^{2,3}$ \& Nélida R. Villaseñor ${ }^{1 *}$
}

${ }^{1}$ Departamento de Gestión Forestal y su Medio Ambiente, Facultad de Ciencias Forestales y de la Conservación de la Naturaleza, Universidad de Chile, La Pintana, Santiago, Chile. ${ }^{2}$ Escuela de Pregrado, Facultad de Ciencias Agronómicas, Universidad de Chile, La Pintana, Santiago, Chile.

${ }^{3}$ Manque Bioexploraciones, La Florida, Santiago, Chile.

*Email: villasenor@uchile.cl

\section{RESUMEN}

El limitado número de investigaciones ecológicas en ecosistemas urbanos de Latinoamérica dificulta manejar las áreas urbanas para la conservación de biodiversidad. Además, la mayoría de los estudios ecológicos en ciudades se han enfocado en parques y plazas, ignorando la mayor parte del ecosistema urbano. Para brindar evidencia científica robusta que permita contribuir al manejo del hábitat urbano para la conservación de aves, investigamos la influencia de variables del hábitat sobre la abundancia de especies de aves nativas y exóticas en la ciudad de Santiago, Chile. Durante la temporada reproductiva de 20172018, evaluamos aves y variables del hábitat en 455 puntos distribuidos a través de la ciudad. Ajustamos modelos lineales generalizados para evaluar la influencia de variables del hábitat sobre la abundancia de las especies de aves. En 905 conteos realizados, registramos 35 especies de aves (31 nativas y 4 exóticas). La abundancia de aves nativas (tórtola, fío-fío, chercán, zorzal, chincol y mirlo) aumentó con la cobertura de vegetación leñosa. Puntos con mayor cobertura arbustiva en el microhábitat exhibieron una mayor abundancia de zorzal, chincol y fío-fío. Opuesto a la respuesta de las aves nativas, la abundancia de las especies exóticas (paloma y gorrión) disminuyó con el aumento de la vegetación. Nuestros resultados demuestran la importancia de la vegetación leñosa para conservar especies de aves nativas en Santiago. Estrategias de manejo que (1) eviten la pérdida de vegetación leñosa e (2) incrementen árboles y arbustos en áreas urbanas carentes de cobertura vegetal promoverían una mayor abundancia de aves nativas.

Palabras clave: avifauna, ecosistemas urbanos, hotspot de biodiversidad, Santiago de Chile, vegetación leñosa.

\begin{abstract}
A paucity of ecological research in urban ecosystems in Latin America limits the management of urban areas for biodiversity conservation. In addition, the majority of previous ecological studies in cities have focused on parks, ignoring most part of urban ecosystems. To provide robust scientific evidence for the management of urban areas for bird conservation, we investigate the influence of habitat variables on the abundance of native and exotic bird species in Santiago, Chile. During the reproductive season of 2017-2018, we surveyed birds and habitat variables at 455 points distributed throughout the city. We fit generalized linear models to evaluate the influence of habitat variables on individual bird species abundance. In 905 bird counts, we recorded 35 bird species (31 native and 4 exotic). The abundance of
\end{abstract}


native birds (Zenaida auriculata, Elaenia albiceps, Troglodytes aedon, Turdus falcklandii, Zonotrichia capensis and Molothrus bonariensis) increased with woody vegetation cover. Points with greater shrub cover in the microhabitat exhibited a greater abundance of T. falcklandii, Z. capensis and E. albiceps. In contrast to the response of native birds, the abundance of exotic species (Columba livia and Passer domesticus) decreased with vegetation cover. Our results demonstrate the importance of woody vegetation to conserve native bird species in Santiago. Management of habitat that (1) avoids the loss of woody vegetation and (2) aims to increase trees and shrubs in urban areas with low vegetation cover will promote a greater abundance of native birds.

Keywords: avifauna, biodiversity hotspot, Santiago of Chile, urban ecosystems, woody vegetation.

\section{INTRODUCCIÓN}

El avance de la ecología urbana en las últimas décadas ha contribuido a comprender la ecología de los organismos que habitan en ecosistemas urbanos. Si bien el número de investigaciones sobre biodiversidad en ecosistemas urbanos ha aumentado considerablemente, la mayoría de los estudios provienen de Europa y Norte América (Wu 2014). América Latina es una de las regiones más urbanizadas y presenta ciudades con fuertes inequidades ambientales, sociales y económicas (MacGregor-Fors \& Escobar-Ibáñez 2017). A pesar de la alta urbanización y la importancia de esta región para la biodiversidad global, los estudios en ecología urbana aún son escasos (Cursach et al. 2012; MacGregor-Fors \& Escobar-lbáñez 2017).

Para conservar biodiversidad en ciudades, en particular fauna, es necesario comprender la relación entre las especies y la estructura del hábitat. Entre los diferentes grupos de animales terrestres, las aves surgen como un buen sujeto de estudio ya que son sensibles a cambios en la estructura y composición del hábitat. Además, son relativamente fáciles de detectar y su evaluación es de bajo costo (Savard et al. 2000).

La vegetación es una de las principales variables que influye sobre la diversidad de aves en ecosistemas urbanos. En particular, la cobertura de árboles, arbustos y herbáceas influiría positivamente sobre la abundancia de aves (Leveau \& Leveau 2004), ya que estas coberturas se asocian con la oferta de alimento, sitios de nidificación, refugio y percha (Silva et al. 2015, Haedo et al. 2017).

Otras coberturas del suelo comunes en los ambientes urbanos, como pavimento y edificaciones, también influencian la abundancia de aves en la ciudad. En general, zonas con una alta densidad de construcción presentan una baja abundancia de aves nativas porque la calidad del hábitat sería baja para estas especies (Haedo et al. 2017; Amaya-Espinel et al. 2019). Sin embargo, algunas especies pueden ser más abundantes en sitios con una mayor densidad de edificaciones (Threlfall et al. 2016, Morelli et al. 2017). Estas especies se clasifican como sinantrópicas y en gran medida corresponden a especies exóticas y cosmopolitas. Su abundancia aumenta desproporcionadamente con la urbanización, reemplazando a las especies nativas a medida que aumenta la superficie construida (McKinney 2006, Chamberlain et al. 2009).

En Chile, existen algunas investigaciones sobre aves en ciudades. Sin embargo, la mayoría de los estudios se han enfocado en parques y plazas, ignorando gran parte del ambiente urbano. En Santiago, capital de Chile y una de las ocho grandes metrópolis en Latinoamérica (MacGregorFors \& Escobar-lbáñez 2017), los estudios de aves se han concentrado en sectores de altos ingresos (sector oriente). Por ejemplo, Estades (1995) describió la relación entre las aves y la vegetación en siete parques de las comunas de Las Condes y Vitacura; mientras que Celis-Diez y colaboradores (2017) evaluaron la riqueza de especies de aves en 10 parques del sector oriente. Díaz \& Armesto (2003) compararon la avifauna presente en diferentes ambientes, incluyendo parques urbanos, barrios residenciales y faldeos precordilleranos en la comuna de La Reina. Entre los estudios, destaca el esfuerzo de muestreo de Varela (2003), quien evaluó el ensamble de aves en las diferentes comunas que conforman la ciudad.

La calidad del hábitat para las aves varía a través de la ciudad debido a que las áreas verdes (i.e. superficie de terreno predominantemente cubierta por vegetación), el arbolado urbano y las edificaciones no se distribuyen de manera homogénea. Por ejemplo, la distribución de las áreas verdes y del arbolado urbano presenta una fuerte inequidad en Santiago: comunas de mayores ingresos tienen mayor superficie y acceso a áreas verdes (Reyes Päcke \& Figueroa 2010), y vecindarios de estratos socioeconómicos altos tienen mayor cantidad y diversidad de árboles que vecindarios de estratos socioeconómicos más bajos (Hernández \& Villaseñor 2018). La densidad de edificaciones también presenta gran variabilidad, fluctuando entre menos de una vivienda por hectárea a más de 1.600 viviendas por hectárea (basado en 
datos por manzana, INE 2019). Por lo tanto, es importante generar información a través de la ciudad para comprender la relación entre las aves y la ciudad de manera integral.

La carencia en Chile de estudios ecológicos a través de la ciudad limita la comprensión de los impactos de la urbanización sobre los ecosistemas, y la habilidad de proponer y tomar decisiones de manejo para la mantención de fauna en ciudades.

Para contribuir a la conservación de aves mediante evidencia científica que permita el manejo del hábitat urbano y la planificación urbana sensible con la biodiversidad, investigamos la influencia de variables del hábitat sobre la abundancia de diferentes especies de aves en la ciudad de Santiago. Específicamente, evaluamos la influencia de la cobertura de vegetación leñosa, arbórea, arbustiva, herbácea, superficies impermeables y edificaciones sobre la abundancia de especies de aves nativas y exóticas. En base a nuestros resultados y la literatura científica, se discuten alternativas de manejo que contribuirán a la conservación de aves en la ciudad.

\section{MATERIALES Y MÉTODOS}

\section{ÁREA DE ESTUDIO}

El estudio se sitúa en la ciudad de Santiago, capital de Chile, ubicada en la zona central del país (Figura 1). Santiago alberga $>6$ millones de habitantes, lo que la posiciona entre las ocho grandes metrópolis de Latinoamérica (MacGregor-Fors \& Escobar-Ibáñez 2017). Presenta $3,7 \mathrm{~m}^{2}$ de áreas verdes por habitante, valor muy por debajo de los $9 \mathrm{~m}^{2}$ por habitante sugeridos por la Organización Mundial de la Salud (Reyes Päcke \& Figueroa 2010). El clima es mediterráneo, con lluvias invernales y un periodo seco prolongado en la época estival (INE 2018). La precipitación media anual es de $312,5 \mathrm{~mm}$ y la temperatura media anual es de $13,9^{\circ} \mathrm{C}$, donde la temperatura máxima se registra en verano $\left(\sim 37^{\circ} \mathrm{C}\right)$, y la temperatura mínima en invierno $\left(\sim-3^{\circ} \mathrm{C}\right.$; INE 2018). La flora y la fauna de Chile central es diversa y presenta un alto nivel de endemismos debido a su aislamiento geográfico (Arroyo et al. 2008). Sin embargo, el paisaje ha sido fuertemente modificado producto del cambio de uso del suelo. Debido al gran número de endemismos junto a la alta pérdida de la vegetación natural, esta área es un "hotspot" de biodiversidad global: un área prioritaria para acciones de conservación (Myers et al. 2010).

\section{SitIOS DE MUESTREO Y PUNTOS DE CONTEO}

Para determinar la zona urbana de la ciudad de Santiago, se consideró el uso "urbano o industrial" en base a un shapefile de usos de suelo del año 2014. Estas áreas comprendieron áreas residenciales, comerciales, industriales y verdes al interior de la ciudad. Este proceso descartó usos de suelo agrícola, pradera, matorral, bosque, humedal y cuerpo de agua. Para evaluar áreas con diferente calidad de hábitat para las aves en la zona urbana, se seleccionaron 118 sitios de muestreo de $1 \mathrm{~km}^{2}$ mediante un muestreo aleatorio estratificado. Los estratos fueron definidos de acuerdo a atributos del arbolado urbano (e.g. cuatro estratos de acuerdo al porcentaje de cobertura arbórea en $1 \mathrm{~km}^{2}$ ), que fueron obtenidos a través de la clasificación de imágenes satelitales de alta resolución (WorldView-2, DigitalGlobe) del año 2014 (datos no publicados). En cada uno de los 118 sitios de muestreo seleccionados en la zona urbana, se establecieron cuatro puntos de conteo. Estos puntos de conteo se localizaron en el árbol/arbusto accesible más cercano a los vértices de un cuadrado de $200 \mathrm{~m}$ de lado, asegurando que las áreas de registro de aves no se sobrepongan (Figura 1a-b).

\section{EVALUACIÓN DE AVES}

Durante la temporada reproductiva (noviembre 2017 a febrero 2018) se realizaron conteos de aves, donde se registró la abundancia de diferentes especies de aves en cada punto de conteo. Para coincidir con la actividad máxima de las aves, los conteos de aves se realizaron durante la mañana 6:0011:30 am) y en días con buenas condiciones meteorológicas. En cada punto de conteo, se registraron todas las aves vistas $\mathrm{u}$ oídas durante 5 minutos en rangos de $10 \mathrm{~m}$ de distancia en una parcela circular de $50 \mathrm{~m}$ de radio (Bibby et al. 2000). En todos los puntos de conteo se realizó una repetición (en un día diferente).

\section{CARACTERIZACIÓN DEL HÁBITAT}

En cada punto de conteo, se calculó el porcentaje de cobertura de vegetación leñosa en parcelas de $50 \mathrm{~m}$ de radio (Figura 1b). Para obtener las coberturas, primero se creó un buffer de $50 \mathrm{~m}$ alrededor de cada punto de conteo. Luego, se digitalizó la cobertura de vegetación leñosa dentro de cada parcela circular mediante fotointerpretación de imágenes satelitales de alta resolución (WorldView, DigitalGlobe, en ArcGIS). En base a los polígonos digitalizados, se calculó el porcentaje de cobertura de vegetación leñosa por parcela (50 m de radio) en ArcGIS.

Para considerar variables a una escala con mayor nivel de detalle, se registraron variables del microhábitat en cada punto de conteo utilizando parcelas de $11 \mathrm{~m}$ de radio (Figura 1b). En cada parcela circular se estimó visualmente, en terreno, el porcentaje de cobertura del estrato herbáceo, arbustivo, arbóreo, de superficies impermeables y de edificaciones (Varela 2003). Parcelas de este tamaño han permitido caracterizar el microhábitat en la ciudad (e.g. Varela 2003; Hernández \& Villaseñor 2018). 


\section{ANÁLISIS DE DATOS}

Previo al análisis estadístico, se exploraron los datos obtenidos. Para cada especie de ave, se graficó su frecuencia de acuerdo a la distancia al observador (0 a 50 m), lo que reveló la dificultad para detectar aves a distancias mayores de $30 \mathrm{~m}$. Por lo tanto, sólo se utilizaron los datos de abundancia hasta $30 \mathrm{~m}$ de radio. Este método fue considerado más apropiado que intentar corregir la abundancia de cada especie por detección imperfecta (e.g. Welsh et al. 2013).

Para evaluar la influencia de las variables del hábitat sobre la abundancia de diferentes especies de aves, sólo se consideraron aquellas especies que presentaron al menos 50 registros y se observaron en al menos el $10 \%$ de los puntos de conteo. Previo a ajustar los modelos, se exploraron las relaciones entre variables y se graficó cada variable respuesta (abundancia de especies de aves) en relación a las variables independientes (variables del hábitat), para observar tendencias y posibles anomalías en los datos.

Para el análisis estadístico, la abundancia de cada especie de ave fue agregada por punto de conteo (i.e. suma de registros del primer conteo y su repetición). Se ajustaron Modelos Lineales Generalizados (log link) que describieron la abundancia de un ave en función de las diferentes variables del hábitat. En todos los modelos se incluyó el logaritmo natural del número de conteos como un "offset", lo que permite considerar diferencias en el esfuerzo de muestreo (en algunos puntos de conteo no fue posible realizar la repetición).

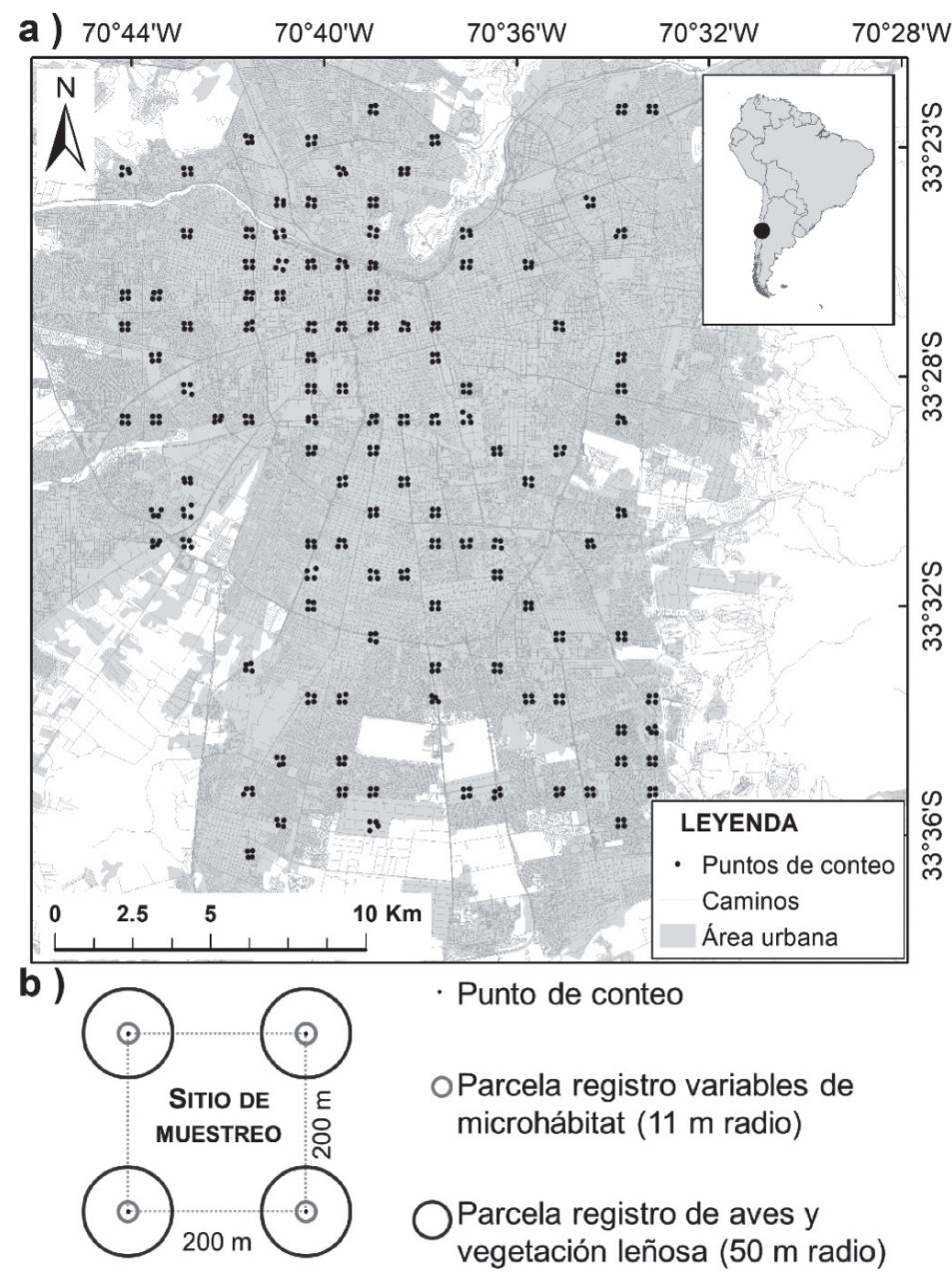

Fıgura 1. (a) Área de estudio en Santiago, Chile, donde se distribuyeron 472 puntos de conteo asociados a 118 sitios de muestreo de $1 \mathrm{~km}^{2}$ en la zona urbana de la ciudad. (b) En cada sitio de muestreo, se establecieron cuatro puntos de conteo, uno en cada vértice de un cuadrado de $200 \mathrm{~m}$ de lado ubicado en el centro del sitio de muestreo. Cada punto de conteo, es el centro de las parcelas para el registro de variables del microhábitat (11 m de radio), y para el registro de aves y de la vegetación leñosa (50 m de radio). / (a) Study area located in Santiago, Chile, where 472 count points were distributed associated to 118 sampling sites ( $\left.1 \mathrm{~km}^{2}\right)$ in the urban area of the city. (b) At each sampling site, four count points were established, one at each vertex of a square (200 m side length) located in the center of the sampling site. Each count point is the plot center for recording microhabitat variables (11 m radius) and for recording birds and woody vegetation (50 $\mathrm{m}$ radius). 
Para las especies con más de 150 registros, los modelos incluyeron todas las variables del hábitat como variables predictivas (seis), además del efecto cuadrático de una variable cuando hubo evidencia de una relación no lineal (Apéndice). Los modelos se ajustaron con distribución de Poisson y con distribución binomial negativa. Ambas distribuciones se utilizan para analizar datos de conteo, donde la última permite captar variación adicional a Poisson (Bolker et al. 2009). Para evitar usar demasiados parámetros en los modelos de aves que presentaron entre 50 a 150 registros, se ajustaron modelos con distintas combinaciones de variables predictivas, permitiendo un máximo de tres variables predictivas por modelo. En estos modelos se utilizó una distribución binomial negativa, ya que presentó un mejor ajuste que Poisson. Para evaluar multicolinealidad, se calculó el valor de inflación de la varianza.

Luego, se seleccionaron los mejores modelos para cada especie de ave. Para las especies con más de 150 registros, se usó el Criterio de Información de Akaike (AIC) (Burnham \& Anderson 2002). Para las especies con menos de 150 registros, se utilizó el Criterio de Información de Akaike corregido para muestras pequeñas ( $\mathrm{AlCc}$ ) con el paquete MuMln (Barton 2019). Finalmente, se exploraron los residuos de los mejores modelos (menor valor de AIC o AICc) para evaluar el ajuste del modelo. Los mejores modelos fueron interpretados y sus intervalos de confianza al $95 \%$ fueron estimados y graficados. Todos los análisis fueron realizados en R 3.5.3 (R Core Team 2019).

\section{RESULTADOS}

Se registró un total de 35 especies de aves, las que corresponden a 31 especies nativas y 4 especies exóticas. En 905 conteos de aves realizados en 455 puntos de conteo (total de puntos de conteo utilizados en el análisis), se registraron 7.044 individuos con una media de 7,79 $\pm 0,26$ (media $\pm \mathrm{EE}$ ) aves por conteo (30 $\mathrm{m}$ de radio). Las especies más abundantes, y que permitieron análisis estadísticos, fueron el gorrión (Passer domesticus) (3,03 $\pm 0,15$ individuos/ conteo), la paloma (Columba livia) $(1,42 \pm 0,19)$, el zorzal (Turdus falcklandii) $(1,09 \pm 0,05)$, la tórtola (Zenaida auriculata) $(0,8 \pm 0,05)$, el chincol (Zonotrichia capensis) $(0,48 \pm 0,04)$, el fío-fío (Elaenia albiceps) $(0,22 \pm 0,02)$, el mirlo (Molothrus bonariensis) $(0,16 \pm 0,02)$ y el chercán (Troglodytes aedon) $(0,13 \pm 0,02)$ (Tabla 1)

\section{VARIABLES DEL HÁBITAT}

La cobertura de vegetación leñosa en las parcelas de $50 \mathrm{~m}$ de radio presentó una media de 15,67\% \pm 0,37 (media \pm
EE). El microhábitat (parcela de $11 \mathrm{~m}$ de radio) fue dominado por superficies impermeables $(54,4 \% \pm 0,99)$, seguido por el estrato arbóreo $(24,72 \% \pm 0,85)$, edificaciones $(14,27 \pm 0,71)$, herbáceo $(11,16 \% \pm 0,7)$ y arbustivo $(3,59 \% \pm 0,25)$ (Tabla 2$)$. Estos porcentajes no deben ser considerados como promedio para la ciudad ya que nuestro diseño experimental establece el centro del punto de conteo asociado un árbol/arbusto. Por lo tanto, el promedio de cobertura arbórea y/o arbustiva en nuestros puntos sería mayor al promedio de la cobertura arbórea y/o arbustiva en la ciudad. Esta diferencia sería mayor a nivel de microhábitat.

\section{MEJORES MODELOS}

Seis especies nativas y dos especies exóticas presentaron al menos 50 registros y se observaron en al menos el $10 \%$ de los puntos de conteo (Tabla 3). De acuerdo a los mejores modelos (identificados por AIC o AICC), la abundancia de estas especies de aves fue mejor modelada con distribución binomial negativa que con Poisson (Apéndice). No hubo evidencia de problemas de multicolinealidad, ya que el factor de inflación de la varianza fue $<2$, exceptuando aquellos modelos donde se utilizó tanto la variable original como su efecto cuadrático.

\section{INFLUENCIA DEL HÁBITAT SOBRE AVES NATIVAS}

La cobertura de vegetación leñosa en parcelas de $50 \mathrm{~m}$ de radio fue la única variable predictiva que presentó un efecto estadísticamente significativo sobre todas las especies de aves nativas (Tabla 3). A medida que la cobertura de vegetación leñosa en parcelas de $50 \mathrm{~m}$ aumentó, incrementó la abundancia de la tórtola, fío-fío, chercán, zorzal, chincol y mirlo en los puntos de conteo (Figura 2a-f). Sin embargo, su efecto no fue lineal sobre las cuatro primeras especies: sus abundancias incrementaron con el aumento de la cobertura de vegetación leñosa hasta que ésta alcanzó un 20-30\% (Tabla 3, Figura 2a-d).

La vegetación a nivel del microhábitat (parcela de 11 $\mathrm{m}$ de radio) también presentó efectos sobre las especies nativas. El aumento de la cobertura arbustiva se asoció a una mayor abundancia del zorzal, chincol y fío-fío en los puntos de conteo (Tabla 3, Figura 2). En el caso de la abundancia del mirlo, la cobertura arbórea en el microhábitat exhibió un efecto negativo (Figura 2f). Respecto a las coberturas no vegetales, un aumento en la cobertura de superficies impermeables se asoció a una menor abundancia del zorzal (Figura 2d), mientras que el aumento de la superficie cubierta por edificaciones se asoció a una menor abundancia de la tórtola (Figura 2a).

INFLUENCIA DEL HÁBITAT SOBRE AVES EXÓTICAS

Opuesto a los resultados en especies nativas, la abundancia 
de especies exóticas (paloma, gorrión) por punto de conteo fue más baja a mayor cobertura de vegetación leñosa (Tabla 3). Además, el aumento de la cobertura arbustiva en el microhábitat se asoció a una menor abundancia del gorrión y la paloma (Figura 2g-h); mientras que una mayor cobertura de superficies impermeables se asoció a una mayor abundancia del gorrión - aunque el efecto no fue lineal (Figura 2h).

TABLA 1. Abundancia promedio (y error estándar) por conteo (30 m de radio) de 35 especies de aves registradas en la ciudad de Santiago. $\left(^{*}\right)$ Especies registradas a más de $30 \mathrm{~m}$ del observador. / Average abundance (and standard error) per count (30 m radius) of 35 bird species recorded in Santiago city. $\left({ }^{*}\right)$ Species recorded at distances larger than $30 \mathrm{~m}$ from the observer.

\begin{tabular}{|c|c|c|c|c|c|}
\hline \multirow{2}{*}{ Familia } & \multirow{2}{*}{ Nombre común } & \multirow{2}{*}{ Nombre científico } & \multirow{2}{*}{ Origen } & \multicolumn{2}{|c|}{ Abundancia } \\
\hline & & & & Promedio & $\mathrm{EE}$ \\
\hline Tinamidae & Perdiz chilena & Nothoprocta perdicaria & Nativa y endémica & $*$ & $*$ \\
\hline \multirow[t]{2}{*}{ Accipitridae } & Águila & Geranoaetus melanoleucus & Nativa & * & $*$ \\
\hline & Aguilucho & Geranoaetus polyosoma & Nativa & * & * \\
\hline Charadriidae & Queltehue & Vanellus chilensis & Nativa & 0,005 & 0,003 \\
\hline Laridae & Gaviota dominicana & Larus dominicanus & Nativa & 0,004 & 0,003 \\
\hline \multirow[t]{4}{*}{ Columbidae } & Paloma & Columba livia & Exótica & 1,42 & 0,19 \\
\hline & Tortolita cuyana & Columbina picui & Nativa & 0,08 & 0,01 \\
\hline & Torcaza & Patagioenas araucana & Nativa & * & $*$ \\
\hline & Tórtola & Zenaida auriculata & Nativa & 0,8 & 0,05 \\
\hline Picidae & Carpinterito & Veniliornis lignarius & Nativa & 0,001 & 0,001 \\
\hline \multirow[t]{4}{*}{ Falconidae } & Halcón peregrino & Falco peregrinus & Nativa & * & $*$ \\
\hline & Cernícalo & Falco sparverius & Nativa & 0,001 & 0,001 \\
\hline & Tiuque & Milvago chimango & Nativa & 0,03 & 0,01 \\
\hline & Cotorra Argentina & Myiopsitta monachus & Exótica & 0,14 & 0,03 \\
\hline Furnariidae & Tijeral & Leptasthenura aegithaloides & Nativa & 0,01 & 0,004 \\
\hline \multirow[t]{3}{*}{ Tyrannidae } & Cachudito & Anairetes parulus & Nativa & 0,03 & 0,007 \\
\hline & Fío-fío & Elaenia albiceps & Nativa & 0,22 & 0,02 \\
\hline & Diucón & Xolmis pyrope & Nativa & 0,003 & 0,002 \\
\hline Cotingidae & Rara & Phytotoma rara & Nativa & 0,04 & 0,007 \\
\hline \multirow[t]{2}{*}{ Hirundinidae } & Golondrina de dorso negro & Pygochelidon cyanoleuca & Nativa & 0,01 & 0,01 \\
\hline & Golondrina Chilena & Tachycineta meyeni & Nativa & 0,03 & 0,01 \\
\hline Troglodytidae & Chercán & Troglodytes aedon & Nativa & 0,13 & 0,02 \\
\hline Turdidae & Zorzal & Turdus falcklandii & Nativa & 1,09 & 0,05 \\
\hline Mimidae & Tenca & Mimus thenca & Nativa & 0,003 & 0,002 \\
\hline \multirow[t]{4}{*}{ Thraupidae } & Diuca & Diuca diuca & Nativa & 0,003 & 0,002 \\
\hline & Cardenal & Paroaria coronata & Exótica & 0,001 & 0,001 \\
\hline & Platero & Phrygilus alaudinus & Nativa & * & * \\
\hline & Chirihue & Sicalis luteola & Nativa & 0,001 & 0,001 \\
\hline Emberizidae & Chincol & Zonotrichia capensis & Nativa & 0,48 & 0,04 \\
\hline \multirow[t]{4}{*}{ Icteridae } & Trile & Agelasticus thilius & Nativa & $*$ & * \\
\hline & Tordo & Curaeus curaeus & Nativa & 0,08 & 0,02 \\
\hline & Mirlo & Molothrus bonariensis & Nativa & 0,16 & 0,02 \\
\hline & Loica & Leistes loyca & Nativa & 0,003 & 0,002 \\
\hline Fringillidae & Jilguero & Spinus barbatus & Nativa & 0,005 & 0,003 \\
\hline Passeridae & Gorrión & Passer domesticus & Exótica & 3,03 & 0,15 \\
\hline
\end{tabular}


TABLA 2. Rango, promedio y error estándar de las variables de hábitat. / Average, range and standard error of habitat variables.

\begin{tabular}{ccccc}
\hline $\begin{array}{c}\text { Escala de medición } \\
\text { (parcela) }\end{array}$ & $\begin{array}{c}\text { Variable } \\
\text { (\% cobertura) }\end{array}$ & Rango (mín.-máx.) & Promedio & EE \\
\hline 50 m de radio & Vegetación leñosa & $0,06-40$ & 15,67 & 0,37 \\
\hline 11 m de radio & Arbórea & $0-95$ & 24,72 & 0,85 \\
& Arbustiva & $0-40$ & 3,59 & 0,25 \\
& Herbácea & $0-90$ & 11,16 & 0,7 \\
& Impermeable & $0-100$ & 54,4 & 0,99 \\
& Edificaciones & $0-70$ & 14,27 & 0,71 \\
\hline
\end{tabular}

a) Z. auriculata
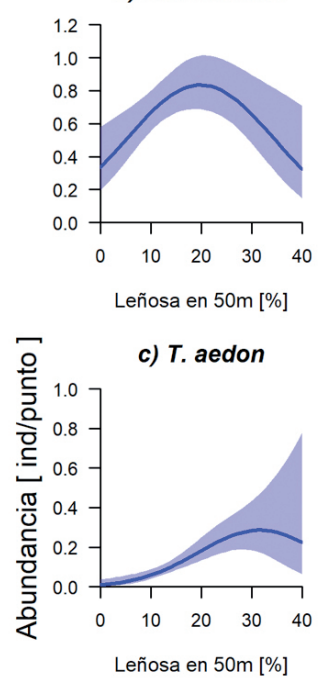

e) Z. capensis

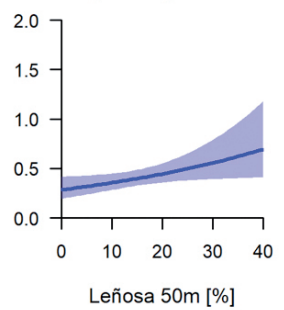

g) C. livia

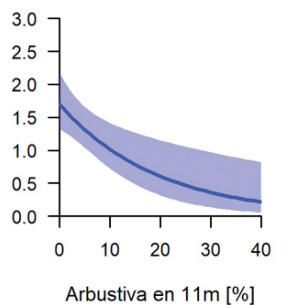

Z. auriculata

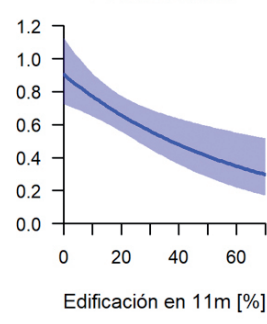

d) T. falcklandii

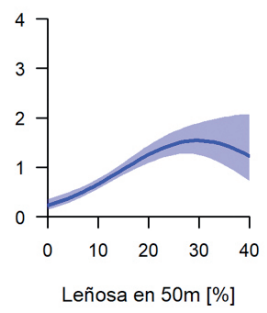

Z. capensis

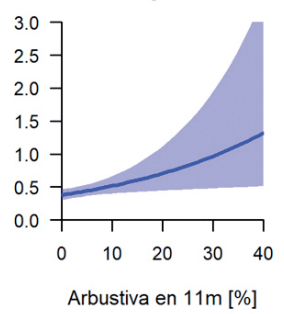

h) P. domesticus

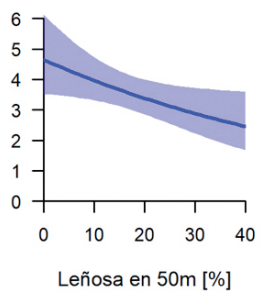

b) E. albiceps

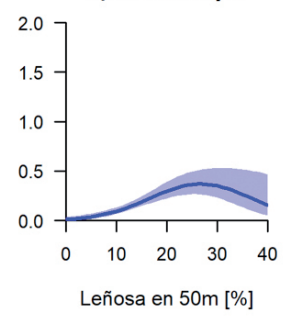

T. falcklandi

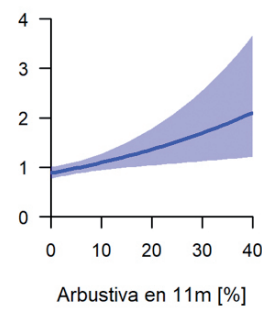

f) $M$. bonariensis

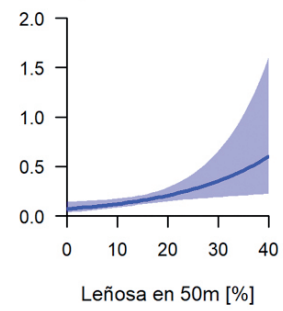

P. domesticus

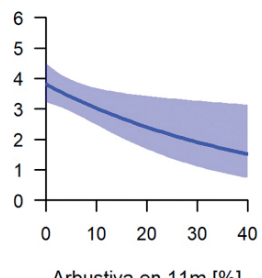

E. albiceps

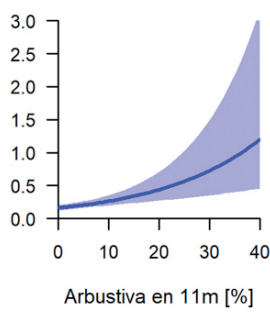

T. falcklandii

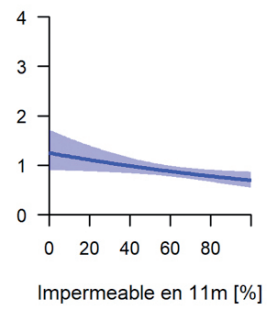

M. bonariensis

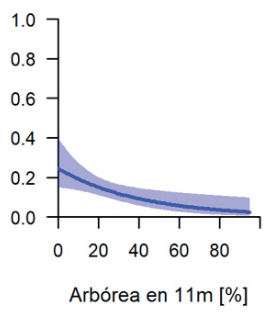

P. domesticus

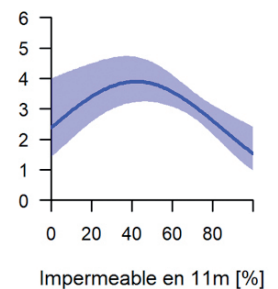

Fıgura 2. Abundancia estimada de ocho especies de aves según variables de hábitat en el mejor Modelo Lineal Generalizado. Abundancia estimada por punto de conteo (30 m de radio) para (a) tórtola (Zenaida auriculata), (b) fío-fío (Elaenia albiceps), (c) chercán (Troglodytes aedon), (d) zorzal (Turdus falcklandii), (e) chincol (Zonotrichia capensis), (f) mirlo (Molothrus bonariensis), (g) paloma (Columba livia), (h) gorrión (Passer domesticus). Áreas sombreadas representan intervalos de confianza al 95\%. / Predicted abundance for eight bird species according to habitat variables in the best Generalized Linear Model. Predicted abundance per count point (30 m radius) for (a) Zenaida auriculata, (b) Elaenia albiceps, (c) Troglodytes aedon, (d) Turdus falcklandii, (e) Zonotrichia capensis, (f) Molothrus bonariensis, (g) Columba livia, (h) Passer domesticus. Shaded areas represent 95\% confidence intervals. 


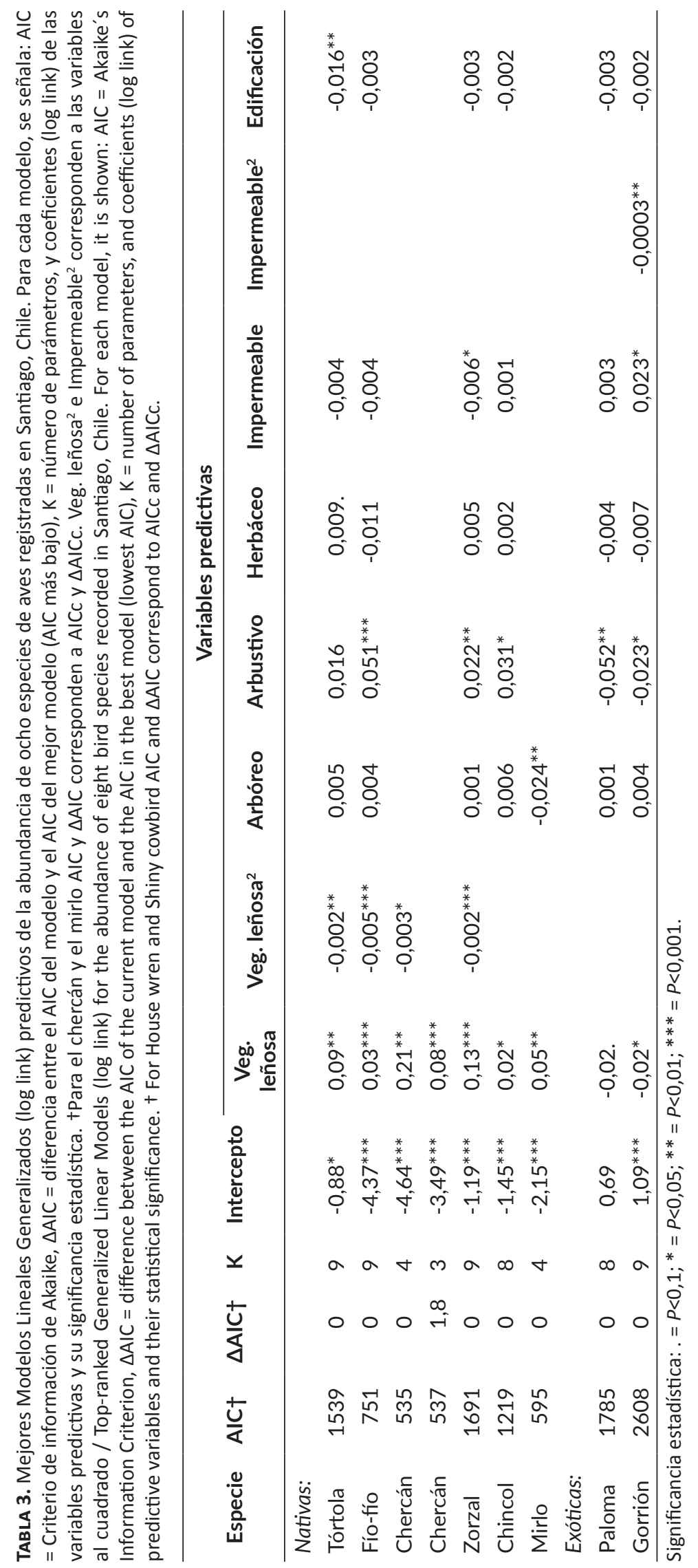




\section{DISCUSIÓN}

El presente estudio provee evidencia científica robusta sobre cómo variables del hábitat urbano influyen en la abundancia de diferentes especies de aves en la ciudad de Santiago, Chile. Nuestros resultados demuestran que la vegetación leñosa es un elemento clave para las especies de aves que habitan en la ciudad. Sin embargo, su efecto varía de acuerdo al origen de las especies: a medida que aumenta la cobertura de vegetación leñosa aumenta la abundancia de especies nativas, y disminuye la abundancia de especies exóticas. A continuación, discutimos nuestros resultados y proponemos estrategias de manejo del hábitat urbano que contribuirían a la conservación de aves nativas en la ciudad.

\section{Aves nATIVAS}

La cobertura de vegetación leñosa promueve una mayor abundancia de aves nativas en Santiago. Este patrón es común en ciudades, donde áreas bien vegetadas presentan una alta riqueza y abundancia de aves (MacGregor-Fors \& Schondube 2011). En Santiago, estudios en áreas verdes han reportado la importancia de la vegetación leñosa sobre la abundancia de aves nativas (e.g. Mella \& Loutit 2007). La vegetación leñosa puede influir de manera positiva sobre diferentes especies de aves nativas, ya que ellas utilizan árboles y arbustos para nidificar, alimentarse y/o buscar refugio (Díaz \& Armesto 2003; Mella \& Loutit 2007).

Sin embargo, la vegetación leñosa presentó valores umbrales sobre los cuales la abundancia de algunas especies nativas comenzaría a disminuir: la abundancia de la tórtola (Zenaida auriculata), el fío-fío (Elaenia albiceps), el chercán (Troglodytes aedon) y el zorzal (Turdus falcklandii) aumentó en los puntos de conteo con el aumento de la cobertura de vegetación leñosa hasta que ésta alcanzó un 20-30\%. Una posible explicación para este patrón es que una cobertura mayor a $20-30 \%$ de vegetación leñosa atraería a una mayor variedad de aves que utilizan árboles y arbustos, aumentando la diversidad y limitando la dominancia de aves comunes del ambiente urbano (McKinney 2006). Además, una alta cobertura de vegetación leñosa podría limitar la abundancia de aves nativas de ambientes mixtos o abiertos. Por ejemplo, el zorzal necesita árboles y arbustos para refugiarse y nidificar (Mella \& Loutit 2007), pero usualmente se le observa forrajeando en praderas de herbáceas (Díaz \& Armesto 2003); mientras que la tórtola frecuentemente forrajea en áreas abiertas, con suelo desnudo (Leveau \& Leveau 2004).

A escala del microhábitat, el fío-fío, el zorzal y el chincol (Zonotrichia capensis) fueron más abundantes a mayor cobertura arbustiva en los puntos de conteo. Contrario a lo esperado, no encontramos un efecto positivo de la cobertura arbustiva sobre la abundancia del chercán, especie que prefiere conglomerados de arbustos para forrajear (Altamirano et al. 2012). Esto sugiere que una parcela de $11 \mathrm{~m}$ de radio no proveería una buena representación del microhábitat para el chercán, siendo necesaria una parcela de mayor tamaño. La abundancia del mirlo fue más baja a mayor cobertura arbórea en el microhábitat, reflejando su hábito de alimentarse en praderas (Martínez \& González 2017).

Una mayor cobertura de superficies impermeables y edificaciones influyó negativamente sobre la abundancia del zorzal y la tórtola, respectivamente. Superficies impermeables y edificaciones compiten con otras coberturas del suelo, afectando a especies que se alimentan en prados, como el zorzal, y en suelos desnudos, como la tórtola. Un desarrollo urbano donde dominan las superficies impermeables y edificaciones afectaría a diferentes especies locales, disminuyendo la riqueza y abundancia de aves nativas (e.g. Silva et al. 2015, Amaya-Espinel et al. 2019).

\section{Aves EXótICAS}

A diferencia de las especies nativas, la abundancia de especies exóticas disminuyó con el aumento de la vegetación. La abundancia de la paloma (Columba livia) y el gorrión (Passer domesticus) fue mayor en puntos de conteo con baja cobertura de vegetación leñosa y/o arbustiva. Estas especies se beneficiarían de una menor cobertura vegetal en áreas urbanas, porque reflejaría el aumento de la superficie construida. Además, la abundancia del gorrión en puntos de conteo fue más alta cuando la cobertura de superficies impermeables alcanzó 40\%. Estas aves son generalistas, omnívoras y sinantrópicas, pueden alimentarse de desechos humanos y tienden a nidificar, refugiarse y posarse en edificaciones e infraestructura urbana (Chamberlain et al. 2009; Leveau \& Leveau 2016).

\section{DIVERSIDAD DE ESPECIES}

Registramos una riqueza total de 35 especies de aves en la ciudad de Santiago durante la temporada estival. Este valor es superior al registrado en Santiago por estudios que se han enfocado en parques y plazas (e.g. Estades 1995 [17 especies]; Celis-Diez et al. 2017 [22]; Amaya-Espinel [28]; Urquiza \& Mella 2002 [31]); pero menor al reportado por trabajos que han evaluado cementerios-parque (Villaseñor \& Escobar 2019 [42]), incluido áreas periurbanas (Muñoz et al. 2018 [39]) y rurales (Díaz \& Armesto 2003 [42]). Respecto a los estudios que han considerado las diferentes comunas que conforman la ciudad de Santiago, registramos una mayor riqueza de especies que Varela (2003) [28] resultado de un mayor esfuerzo de muestreo en nuestro estudio; pero una menor riqueza que Gutiérrez-Tapia et al. (2018) [46], 
quienes utilizaron ocho años de registros de aves en eBird. Debido a que nuestro estudio sólo comprendió la época estival, una mayor riqueza de especies se reportará al incluir evaluaciones durante la temporada no reproductiva (otoño e invierno). En la temporada no reproductiva, diferentes especies de aves migran e ingresan a la ciudad de Santiago, como el picaflor chico (Sephanoides sephaniodes), la dormilona tontito (Muscisaxicola macloviana), la viudita (Colorhamphus parvirostris), entre otras (Villaseñor \& Escobar 2019).

Las especies más abundantes fueron el gorrión y la paloma, ambas exóticas. La dominancia de estas especies es común en ambientes urbanos (Marzluff et al. 2001, Silva et al. 2015). Las aves nativas más abundantes (e.g. zorzal, chincol, tórtola, mirlo) corresponden a especies mayoritariamente generalistas, que habitan en ambientes urbanos y comunes en otras ciudades de Chile (e.g. Concepción, Soto 2014; Valdivia, Silva et al. 2015) y América Latina (e.g. Argentina, Juri \& Chani 2009; Perú, Luque et al. 2018). El ensamble de aves nativas fue dominado por especies del matorral esclerófilo, siendo escasos los registros de especies nativas especialistas de hábitat o que prefieren áreas más boscosas. Las aves rapaces también presentaron una baja abundancia (Tabla 1) debido a que son depredadores tope y la expansión urbana afectaría a diferentes especies (Alvarado et al. 2015). Sin embargo, el tiuque (Milvago chimango) es un ave rapaz común en la ciudad. El tiuque es un omnívoro facultativo que consume alimentos disponibles en el ambiente (Tobar et al. 2014), incluyendo deshechos humanos (Alvarado et al. 2015).

A pesar del gran esfuerzo de muestreo, sólo registramos una especie endémica: la perdiz chilena (Nothoprocta perdicaria); y una cuasi endémica: la tenca (Mimus thenca). La actual matriz urbana sería inhóspita para otras aves endémicas de la zona mediterránea de Chile central, como el tapaculo (Scelorchilus albicollis), la turca (Pteroptochos megapodius), el churrín del norte (Scytalopus fuscus) y el canastero (Pseudasthenes humicola). La carencia de registros de aves endémicas señala que la urbanización es una amenaza para las especies endémicas de este "hotspot" de biodiversidad.

\section{RECOMENDACIONES PARA EL MANEJO DEL HÁBITAT URBANO}

El manejo del hábitat urbano es clave para la conservación de la biodiversidad en ciudades (Aronson et al. 2017). Nuestros resultados demuestran la importancia de la vegetación leñosa (árboles y arbustos) en la ciudad para mantener una alta abundancia de aves nativas y limitar la abundancia de especies exóticas (paloma y gorrión). Por lo tanto, para contribuir a la conservación de aves nativas, es recomendable: (1) mantener árboles y arbustos existentes, y (2) aumentar árboles y arbustos en áreas de baja cobertura.

(1) Mantener la cobertura de árboles y arbustos existentes.
Medidas de mitigación de proyectos inmobiliarios que involucren el reemplazo de árboles adultos por árboles juveniles, no compensarían la pérdida de servicios ecosistémicos brindados por los árboles añosos (Stagoll et al. 2012, Morelli et al. 2017). Árboles añosos promoverían una mayor cantidad de aves nidificantes (Altamirano et al. 2012), mayor riqueza y abundancia de aves, y mayor riqueza de especies asociadas a bosques (Stagoll et al. 2012). Por lo tanto, proyectos inmobiliarios deberían considerar mantener el mayor número posible de árboles existentes e incorporarlos a las áreas verdes o vegetación vial proyectadas. Si árboles adultos serán eliminados, se debería explorar la factibilidad de su rescate y traslado a áreas de baja cobertura en la ciudad.

La poda también es una medida de manejo importante, ya que cuando es excesiva y masiva, disminuye notablemente la cobertura arbórea y arbustiva afectando la oferta de recursos (e.g. alimento, refugio). Si se realiza durante la época reproductiva puede afectar el éxito reproductivo de las aves (e.g. la exposición de nidos aumentaría el riesgo de depredación). Es recomendable que la poda se realice durante el invierno, que no elimine más de un cuarto del área foliar de las plantas leñosas, y se focalice sobre las partes problemáticas (e.g. ramas peligrosas o que dificulten la visión) (Purcell 2015).

(2) Aumentar árboles y arbustos en áreas de baja cobertura. Vecindarios con baja cobertura vegetal deberían ser prioridad en programas de arborización. En Santiago, la distribución de las áreas verdes y del arbolado urbano presenta una evidente inequidad. Debido a que la abundancia de aves se asocia a la cobertura vegetal, áreas de menores ingresos no sólo presentarían menor superficie de áreas verdes y cobertura del arbolado urbano (Reyes Päcke \& Figueroa 2010, Hernández \& Villaseñor 2018), sino también una menor abundancia de aves nativas, acentuando la inequidad en el acceso a la naturaleza en la ciudad.

Finalmente, debido a la importancia del "hotspot" de Chile central para la biodiversidad global, es necesario fomentar la investigación y el desarrollo de una silvicultura urbana basada en plantas nativas adaptadas a las condiciones locales, junto con evitar la pérdida de la vegetación natural.

\section{CONCLUSIÓN}

Nuestro estudio demuestra que la vegetación leñosa es un elemento clave para la abundancia de diferentes especies de aves en Santiago. La vegetación leñosa influye positivamente sobre la abundancia de aves nativas, y limita la abundancia de aves exóticas (paloma y gorrión). Estrategias de manejo del hábitat urbano que mantengan la cobertura arbórea y arbustiva, y aumenten árboles y arbustos en barrios de baja 
cobertura vegetal contribuirán a la conservación de aves nativas en la ciudad.

\section{AGRADECIMIENTOS}

Los autores agradecen el financiamiento de CONICYT FONDECYT Postdoctorado No. 3170179 "Conservation in the city" (IR: N. Villaseñor). Además, agradecen participación en toma de datos: J. Foncea, C. Muñoz, J. Rodríguez, P. Fernández, V. Olivares y M. Carrasco; en clasificación y mapeo de la cobertura arbórea: M. Quintanilla; y fotointerpretación de imágenes: N. Parra y L. Rojas.

\section{REFERENCIAS}

Altamirano, T., Ibarra, J., Hernández, F., Rojas, I., Laker, J., Bonacic, C. 2012. Hábitos de nidificación de las aves del bosque templado andino de Chile. Serie Fauna Australis, Facultad de Agronomía e Ingeniería Forestal, Pontificia Universidad Católica de Chile, Santiago. 113 pp.

Alvarado, S.A., Figueroa, R., Valladares Faundez, P., CarrascoLagos, P., Moreno, R.A. 2015. Aves Rapaces de la Región Metropolitana de Santiago, Chile. Universidad de Chile, Facultad de Ciencias Forestales y Conservación de la Naturaleza, Santiago. 132 pp.

Amaya-Espinel, J.D., Hostetler, M., Henríquez, C., Bonacic, C. 2019. The influence of building density on Neotropical bird communities found in small urban parks. Landscape and Urban Planning 190:103578.

Aronson, M.F., Lepczyk, C.A., Evans, K.L., Goddard, M.A., Lerman, S.B., Maclvor, J.S., Nilon, C.H., Vargo, T. 2017. Biodiversity in the city: Key challenges for urban green space management. Frontiers in Ecology and the Environment 15(4):189-196.

Arroyo, M., Marquet, P., Marticorena, C., Simonetti, J., Cavieres, L., Squeo, F., Rozzi, R., Massardo, F. 2008. El hotspot chileno, prioridad mundial para la conservación. En: Rovira, J., Ugalde, J., Stutzin, M. (Eds) Biodiversidad de Chile, patrimonio y desafíos: 95-98. Comisión Nacional del Medio Ambiente, Santiago.

Barton, K. 2019. MuMIn: Multi-Model Inference.

Bibby, C.J., Burgess, N.D., Hill, D.A., Mustoe, S.H. 2000. Birds census techniques. Academic press, London. 302pp.

Bolker, B.M., Brooks, M.E., Clark, C.J., Geange, S.W., Poulsen, J.R., Stevens, M.H.H., White, J.S.S. 2009. Generalized linear mixed models: a practical guide for ecology and evolution. Trends in Ecology and Evolution 24(3): 127-135.

Burnham, K. P., Anderson, D.R. 2002. Model Selection and
Multimodel Inference. Springer-Verlag, New York. 488 pp. Celis-Diez, J.L., Muñoz, C.E., Abades, S., Marquet, P.A., Armesto, J.J. 2017. Biocultural homogenization in urban settings: Public knowledge of birds in city parks of Santiago, Chile. Sustainability (Switzerland) 9(485): 1-14.

Chamberlain, D.E., Cannon, A.R., Toms, M.P., Leech, D.I., Hatchwell, B.J., Gaston, K.J. 2009. Avian productivity in urban landscapes: a review and meta-analysis. Ibis 151: 1-18.

Cursach, J.A., Rau, J.R., Tobar, C.N., Ojeda, J.A. 2012. Estado actual del desarrollo de la ecología urbana en grandes ciudades del sur de Chile. Revista de Geografia Norte Grande 52: 57-70.

Díaz, I.A., Armesto, J.J. 2003. La conservación de las aves silvestres en ambientes urbanos de Santiago. Revista Ambiente y Desarrollo de CIPMA 19(2): 31-38.

Estades, C.F. 1995. Aves y vegetacion urbana: el caso de las plazas. Boletín Chileno de Ornitología 2: 7-13.

Gutiérrez-Tapia, P., Azócar, M.I., Castro, S.A. 2018. A citizenbased platform reveals the distribution of functional groups inside a large city from the Southern Hemisphere: e-Bird and the urban birds of Santiago (Central Chile). Revista Chilena de Historia Natural 91(3): 1-16.

Haedo, J., Gioia, A., Aráoz, E., Paolini, L., Malizia, A. 2017. Primary productivity in cities and their influence over subtropical bird assemblages. Urban Forestry \& Urban Greening 26: 57-64.

Hernández, H.J., Villaseñor, N.R. 2018. Twelve-year change in tree diversity and spatial segregation in the Mediterranean city of Santiago, Chile. Urban Forestry \& Urban Greening 29: $10-18$

Instituto Nacional de Estadística (INE). 2018. Informe anual de medioambiente. INE, Santiago. 172pp.

Instituto Nacional de Estadística (INE). 2019. Densidad de Vivienda Urbana 2017: Región Metropolitana de Santiago. Geodatos Abiertos INE. URL: http://geoine-ine-chile. opendata.arcgis.com. Accedido: Octubre 4, 2019.

Juri, M.D., Chani, J.M. 2009. Variación estacional en la composición de las comunidades de aves en un gradiente urbano. Ecologia Austral 19: 175-184.

Leveau, L.M., Leveau, C.M. 2004. Comunidades de aves en un gradiente urbano de la ciudad de Mar del Plata, Argentina. Hornero 19(1): 13-21.

Leveau, L.M., Leveau, C.M. 2016. Does urbanization affect the seasonal dynamics of bird communities in urban parks? Urban Ecosystems 19: 631-647.

Luque , C.R., Cano, L.G., Peña, Y.A. 2018. Richness and abundance of birds in an urban gradient of Arequipa, southwest of Peru. Arnaldoa 25: 1095-1106.

MacGregor-Fors, I., Schondube, J.E. 2011. Gray vs. green 
urbanization: Relative importance of urban features for urban bird communities. Basic and Applied Ecology 12: 372-381.

MacGregor-Fors, I., Escobar-Ibáñez, J.F., 2017. Avian Ecology in Latin American Cityscapes. Springer. 173pp.

Martínez, D., G. González. 2017. Aves de Chile: guía de campo y breve historia natural. Ediciones del Naturalista. Santiago. 540 pp.

Marzluff, J.M., Bowman, R., Donnelly, R. 2001. A historical perspective on urban bird research: trends, terms, and approaches. En: Marzluff J.M., Bowman R., Donnelly R. (Eds) Avian Ecology and Conservation in an Urbanizing World: 1-17. Springer, Boston.

McKinney, M.L. 2006. Urbanization as a major cause of biotic homogenization. Biological Conservation 127: 247-260.

Mella, J.E., Loutit, A. 2007. Ecologia comunitaria y reproductiva de aves en cerros islas y parques de Santiago. Boletín Chileno de Ornitología 13: 13-27.

Morelli, F., Benedetti, Y., Su, T., Zhou, B., Moravec, D., ?ímová, P., Liang, W. 2017. Taxonomic diversity, functional diversity and evolutionary uniqueness in bird communities of Beijing's urban parks: Effects of land use and vegetation structure. Urban Forestry and Urban Greening 23: 84-92.

Muñoz, C.E., Undurraga, M.I., Saratschef, T., Rannou, T., CelisDiez, J. 2018. Diversidad y conocimiento de las aves urbanas por habitantes de Santiago, Chile. En: Figueroa, J., Lazzoni, I. (Eds) Biodiversidad urbana en Chile: Estado del arte y los desafíos futuros: 283-315. Ediciones Universidad Central de Chile, Santiago.

Myers, N., Mittermeier, R.A., Mittermeier, C.G., da Fonseca, G.A., Kent, J. 2010. Biodiversity hotspots for conservation priorities. Nature 403: 853-858.

Purcell, L. 2015. Lo esencial para la poda de árboles. Forestry and Natural Resources 506: 1-18.

R Core Team. 2019. R: A language and environment for statistical computing. R Foundation for Statistical Computing, Vienna, Austria. URL: https://www.R-project.org/.

Reyes Päcke, S., Figueroa , I. 2010. Distribución, superficie y accesibilidad de las áreas verdes en Santiago de Chile. Revista Latinoamericana de Estudios Urbanos Regionales 36: 89-110.
Savard, J.L., Clergeau, P., Mennechez, G. 2000. Biodiversity concepts and urban ecosystems. Landscape and Urban Planning 48: 131-142.

Silva, C.P., García, C.E., Estay, S.A., Barbosa, O. 2015. Bird richness and abundance in response to urban form in a Latin American City: Valdivia, Chile as a case study. PLoS ONE 10(9): 1-16.

Soto, R. 2014. Efectos del grado de urbanización sobre la comunidad de aves en la ciudad de Concepción, VIII Región, Chile. Magister en Ciencias mención Zoología. Facultad de Ciencias Naturales y Oceanográficas, Universidad de Concepción. Chile, Concepción. 42 pp.

Stagoll, K., Lindenmayer, D.B., Knight, E., Fischer, J., Manning, A.D. 2012. Large trees are keystone structures in urban parks. Conservation Letters 5: 115-122.

Threlfall, C.G., Williams, S.G., Hahs, A.K., Livesley, S.J. 2016. Approaches to urban vegetation management and the impacts on urban bird and bat assemblages. Landscape and Urban Planning 153: 28-39.

Tobar, C., Rau, J., Santibáñez, A., Arriagada, A., Sade, S., Araneda, R., Tello, F. 2014. Diet of the Chimango Caracara (Milvago chimango) in agroecosystems of Osorno city, southern Chile. Boletín Chileno de Ornitología 20(1-2): 13-16.

Urquiza, A., Mella, J. 2002. Riqueza y diversidad de las aves en parques de Santiago durante el período estival. Boletín Chileno de Ornitología 9: 12-21.

Varela, S. 2003. Calidad de la vegetación urbana como hábitat para aves. El caso de Santiago de Chile. Ingeniero Forestal. Facultad de Ciencias Forestales, Universidad de Chile. Chile, Santiago. 116 pp.

Villaseñor, N.R., Escobar, M.A.H. 2019. Cemeteries and biodiversity conservation in cities: how do landscape and patch-level attributes influence bird diversity in urban park cemeteries? Urban Ecosystems 22(6): 1037-1046.

Welsh, A.H., Lindenmayer, D.B., Donnelly, C.F. 2013. Fitting and interpreting occupancy models. PLoS ONE 8(1): 1-21.

Wu, J. 2014. Urban ecology and sustainability: The state-ofthe-science and future directions. Landscape and Urban Planning 125: 209-221.

\section{APÉNDICE}

Selección de los mejores Modelos Lineales Generalizados para la abundancia de ocho especies de aves. Puede ser descargado desde: http://dx.doi.org/10.17632/kwt427y4b5.2

Received: 08.06.2019

Accepted: 14.10.2019 